\title{
Minimization of the Drug and Gene Interactions in Polypharmacy Therapies Augmented with COVID-19 Medications
}

\author{
Azra LAGUMDZIJA-KULENOVIC ${ }^{\mathrm{a}, 1}$ and Adnan KULENOVIC ${ }^{\mathrm{a}}$ \\ a Absolute Information Age, Inc. Toronto, Canada
}

\begin{abstract}
Medications Dexamethasone, Remdesivir or Colchicine, used to treat COVID-19 patients, have significant interactions with other medications and the human genome. The study presented in this paper investigates how to use the Personalized Medicine Therapy Optimization Method (PM-TOM) to minimize these interactions in polypharmacy therapies of COVID-19 patients. We applied PM-TOM on the EMR database of Harvard Personal Genome Project (PGP), drug database DrugBank and Comprehensive Toxicogenomics Database (CTD) to analyze polypharmacy therapies augmented with these medications. The main finding is that these COVID-19 medications significantly increase the drug and gene interactions in partially optimized (or unoptimized) therapies, which is not the case in the fully optimized ones. For example, the test results show that in polypharmacy treatments for patients having between 3 and 8 conditions, the average number of drug and gene interactions in partially optimized therapies ranges from 3 to 18 after adding Remdesivir, 4.3 to 20 Colchicine, and 4.7 to 23 Dexamethasone. On the other hand, these interactions in fully optimized therapies range only 0.6 to $5.2,1.2$ to 7 , and 2.7 to 11 , respectively. These results suggest that polypharmacy therapies should be carefully examined before adding these medications. This recommendation applies to all other situations when polypharmacy patients may conduct new serious conditions, such as COVID-19, requiring additional medications with a high number of drug and gene interactions.
\end{abstract}

Keywords. COVID-19, PM-TOM, Clinical Decision Support Systems (CDSS), Dexamethasone, Remdesivir, Colchicine, Polypharmacy, Multimorbidity, DrugDrug Interactions $(D D I)$, Drug-Gene Interactions $(D G I)$.

\section{Introduction}

The Medical Guidelines for Treating COVID-19 Patients [1] of the US National Institutes of Health (NIH) recommend drugs for various categories of these patients. For example, high-risk hospitalized patients who do not require supplemental oxygen should be treated with Remdesivir. Dexamethasone is recommended only for patients who need invasive mechanical ventilation or extracorporeal membrane oxygenation (ECMO). NIH also reported several active or completed studies on the benefits of Colchicine for COVID-19 patients. For example, study [2] found that the median hospitalization time was reduced from nine to seven days for the COVID-19 patients treated with Colchicine.

\footnotetext{
${ }^{1}$ Corresponding Author, Azra Lagumdzija-Kulenovic, Absolute Information Age, Inc. Toronto, Canada; E-mail: azra.kulenovic@rogers.com.
} 
While these medications could help the COVID-19 patients to overcome this infection without serious outcomes, they may also pose a significant risk due to their numerous drug-drug interactions and drug-gene interactions (DDIs, DGls). For example, the total number of DDIs for Dexamethasone is 1,211, Colchicine 1,297 and Remdesivir 442, according to DrugBank [3].

Several studies, for example [4], report a high occurrence of adverse drug and gene interactions in polypharmacy cases (66-87\% of the patients had DDIs and $34-73 \%$ DGIs). In addition, study [5] found that adverse drug reactions and interactions were between the fourth and sixth causes of death in the US. So, the possible adverse drug and gene interactions of the COVID-19 medications should be carefully considered before adding them to polypharmacy treatment of multimorbidity patients infected with $S A R S-C o V-2$.

This study used the Personalized Medicine - Therapy Optimization Method (PM$T O M)[6,7]$ to optimize $D D I s$ and $D G / s$ in polypharmacy treatments extended with the COVID-19 medications. To the best of our knowledge, PM-TOM is the only published method for optimizing both the drug-drug and drug-gene interactions in polypharmacy treatments. The results suggest that these interactions can be significantly reduced, thus increasing the chances for multimorbidity patients to overcome COVID-19 with minimal consequences.

\section{PM-TOM Method}

The PM-TOM datasets are a database of Electronic Medical Records (EMR), Drug Repository (DRG), and Gene-Drug Repository (GDR). PM-TOM retrieves the patient's conditions, prescriptions and genome from $E M R$ (only pharmacogenetic and pathogenic gene variations). The drug repository (DRG) offers data about the active medication ingredients (referred to as "drugs" in this paper), commercial drug products (referred to as "drug products") and drug-drug interactions. Gene-Drug Repository (GDR) keeps data about drug-gene interactions and their severities. Further information about the PM-TOM Data Model can be found in papers [6, 7].

PM-TOM inputs are a patient's: conditions, genome and drug products considered by a physician, clinician or clinical pharmacist for treating these conditions. Several drug products can be provided for each condition, with an indication of the preferred one. The list of preferred drug products forms so-called Initial Therapy (IT).

PM-TOM outputs are (i) Optimal therapy for considered drugs (OTCD), (ii) Optimal therapy for all drugs (OTAD) and (iii) a Personalized Therapy Report (PTR). PM-TOM creates $O T C D$ after examining drug and gene interactions in the considered drugs and OTAD after looking into all drugs in EMR prescribed for the same conditions by other physicians, clinicians or clinical pharmacists. In the rest of this paper, OTCD will also be referred to as partially optimized therapy and OTAD as fully optimized therapy. Lastly, PTR reports the patient's demographic data, conditions, considered drugs and drug products, partially and fully optimized therapies, along with their Cumulative Adverse Drug Interactions Indicators (CADI), explained below.

As the number of all combinations of potential medications for a patient's conditions could be rather high, $P M-T O M$ implements an iterative heuristic algorithm [6] that examines a select set of candidate therapies rather than all possible ones. For finding an OTCD, these candidate therapies are derived from the initial therapy (IT) by replacing only one drug preferred for a condition with another drug considered for the 
same condition. For creating the corresponding OTAD, the PM-TOM would repeat the same process, starting with $O T C D$ rather than $I T$ and examining all medications from EMR indicated for the same conditions.

For each candidate therapy, $P M-T O M$ calculates indicator $C A D I$ as a weighted sum of its drug-drug and drug-gene interactions $[6,7]$. The optimal therapy will be formed by selecting a drug for each condition whose candidate therapy has minimal CADI against the candidate therapies formed from other drugs considered for the same condition. In that way, the algorithm evaluates only a small subset of all candidate therapies, which significantly reduces its complexity.

\section{Testing Results and Discussion}

In this study, we implemented the PM-TOM method on 484 EMRs from the PGP database of the Harvard Personal Genome Project [8]. These records include the patient's conditions, drugs prescribed for the treatment of these conditions and genome. In addition, the drug database DrugBank [3] was used to find the drug-drug interactions (DDIs) and the Comprehensive Toxicogenomics Database (CTD) [9] to retrieve druggene interactions (DGIs).

We added condition COVID-19 and then medications Dexamethasone, Remdesivir or Colchicine to the PGP EMRs. Then we used PM-TOM to calculate the CADIs of their OTCD and OTAD therapies before adding each of these medications and after. Table 1 summarizes information about the groups of examined EMRs: the number of conditions before the patient contracted COVID-19 (Conditions\#), the total number of patients in each group (Cases\#), the average number of patients' pharmacogenetic and pathological genes (Genes\#) and the average number of all drugs from EMR indicated for patient's conditions (Drugs\#). It also shows the average CADIs of OTCDs and OTADs in the original therapies and therapies augmented with each COVID-19 medication.

Table 1. Testing cases and their drug and gene interaction indicator (CADI) before and after applying COVID19 medications. OTCD-Optimal Therapy for Considered Drugs, OTAD-Optimal Therapy for All Drugs.

\begin{tabular}{|c|c|c|c|c|c|c|c|c|c|c|c|}
\hline 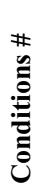 & 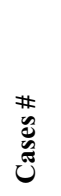 & 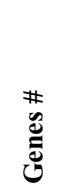 & 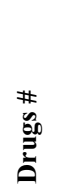 & 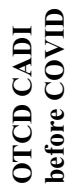 & 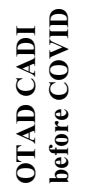 & 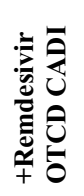 & 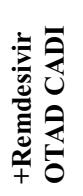 & 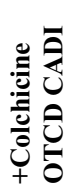 & 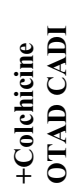 & 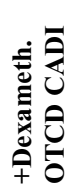 & 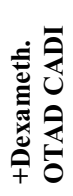 \\
\hline 1 & 272 & 5.4 & 9.6 & 0.2 & 0.03 & 0.5 & 0.06 & 1.0 & 0.33 & 2.8 & 2.2 \\
\hline 2 & 122 & 7.4 & 18.5 & 1.0 & 0.3 & 1.7 & 0.4 & 2.4 & 0.7 & 4.7 & 3.2 \\
\hline 3 & 49 & 3.9 & 28.5 & 2.1 & 0.4 & 3.0 & 0.6 & 4.3 & 1.2 & 5.5 & 2.7 \\
\hline 4 & 16 & 4.4 & 28.7 & 3.4 & 1.1 & 4.9 & 1.6 & 6.1 & 2.7 & 7.6 & 4.4 \\
\hline 5 & 13 & 4.5 & 41.8 & 4.9 & 2.1 & 6.6 & 2.7 & 8 & 3.5 & 10.2 & 6.1 \\
\hline 6 & 3 & 0 & 49.7 & 8.3 & 5.3 & 10.3 & 5.7 & 12.7 & 7 & 12.3 & 7 \\
\hline $7-8$ & 5 & 6 & 61.8 & 15.4 & 5 & 18 & 5.2 & 20.4 & 7 & 23.2 & 11 \\
\hline$>8$ & 4 & 15.5 & 49.5 & 24.2 & 19.5 & 25.2 & 21.2 & 31.2 & 29 & 34 & 28 \\
\hline
\end{tabular}

These results confirm previous findings that $P M-T O M$ can find polypharmacy treatments with significantly reduced drug and gene interactions. For example, in the 
group of three conditions, the average OTCD's CADI is 3.0 after adding Remdesivir, while the average OTAD's CADI is only 0.6. For Colchicine, these reductions are from 4.3 to 1.2 and Dexamethasone from 5.5 to 2.7. In the group with five conditions, these decreases are even more substantial: 6.6 to $2.7,8$ to 3.5 and 10.2 to 6.1 .

The second observation is that these COVID-19 medications significantly increase the drug and gene interactions in partially optimized therapies and, consequently, unoptimized ones, which is not the case in fully optimized therapies. For example, as shown in Table 1 , in polypharmacy patients having 3 to 8 conditions, the CADIs of partially optimized therapies including Remdesivir would increase from 3 (in 3 conditions) to 18 (in 8 conditions), for Colchicine from 4.3 to 20 and Dexamethasone 4.7 to 23 . On the other hand, the $C A D I$ of the fully optimized treatments ranges only from 0.6 to $5.2,1.2$ to 7 , and 2.7 to 11 , respectively.

\section{Conclusion}

These results further emphasize a need for the minimization of adverse drug and gene interactions in polypharmacy therapies. The above findings also show significant room for further fine-tuning of these therapies should a physician, clinician, or clinical pharmacist opt to modify $P M-T O M$ recommendations due to the aspects not currently considered in this method, such as patient's age, gender, ethnicity, etc.

Proper selection of polypharmacy therapies is particularly important when the polypharmacy patients could acquire new severe conditions, such as COVID-19, which may need medications with a high number of drug and gene interactions, like Dexamethasone or Remdesivir or Colchicine.

\section{References}

[1] COVID-19 Treatment Guidelines Panel. Coronavirus Disease 2019 (COVID-19). National Institutes of Health (NIH).

[2] Lopes MI, Bonjorno LP, Giannini MC, et al. Beneficial effects of Colchicine for moderate to severe COVID-19: A Randomized, Double-blinded, Placebo-controlled clinical trial. RMD Open 2021;7: e001455. doi:10.1136/rmdopen-2020-001455

[3] Wishart DS, Feunang YD, Guo AC, Lo EJ, Marcu A, Grant JR, Sajed T, Johnson D, Li C, Sayeeda Z, Assempour N. DrugBank 5.0: a major update to the DrugBank database for 2018. Nucleic acids research. 2018 Jan 4;46(D1):D1074-82.

[4] Reynolds KK, Pierce DL, Weitendorf F, Linder MW. Avoidable drug-gene conflicts and polypharmacy interactions in patients participating in a personalized medicine program. Personalized Medicine. 2017; 14:221-233.

[5] Lazarou J, Pomeranz BH, Corey PN. Incidence of adverse drug reactions in hospitalized patients: a metaanalysis of prospective studies. JAMA 1998;15:1200-1205.

[6] Kulenovic A, Lagumdzija-Kulenovic A. PM-TOM: A method for finding personalized polypharmacy therapies with minimal adverse drug-drug, drug-gene and drug-condition interactions. InDigital Personalized Health and Medicine 2020 (pp. 648-652). IOS Press.

[7] Kulenovic A, Lagumdzija-Kulenovic A. Using PM-TOM for the Minimization of Adverse Drug and Gene Interactions in Therapies for Common Multi-Diseases. Studies in Health Technology and Informatics. 2020 Jun 1;272:205-8.

[8] Ball MP, Bobe JR, et al. Harvard Personal Genome Project: lessons from participatory public research. Genome medicine. 2014 Feb;6(2):1-7.

[9] Davis AP, Grondin CJ, Johnson RJ, Sciaky D, McMorran R, Wiegers J, Wiegers TC, Mattingly CJ. The comparative toxicogenomics database: update 2019. Nucleic acids research. 2019 Jan 8;47(D1):D94854. 